\title{
(GIGA)bYte
}

\section{The draft genome assembly of the critically endangered $N y s s a$ yunnanensis, a plant species with extremely small populations endemic to Yunnan Province, China}

\author{
Weixue $\mathrm{Mu}^{1, *}$, Jinpu $\mathrm{Wei}^{1}$, Ting Yang ${ }^{1}$, Yannan Fan ${ }^{1}$, Le Cheng ${ }^{2}$, \\ Jinlong Yang ${ }^{2}$, Ranchang $\mathrm{Mu}^{3}$, Jie $\mathrm{Liu}^{3}$, Jianming Zhao ${ }^{3}$, Weibang Sun ${ }^{4}$, \\ Xun $\mathrm{Xu}^{1,7}$, Xin Liu ${ }^{1}$, Radoje Drmanac ${ }^{1,6}$ and Huan Liu ${ }^{1,5}$ \\ 1 State Key Laboratory of Agricultural Genomics, BGI-Shenzhen, Shenzhen 518083, China \\ 2 BGI-Yunnan, BGI-Shenzhen, Kunming, 650106, China \\ 3 Forestry Bureau of Ruili, Yunnan Dehong, Ruili 678600, China \\ 4 Yunnan Key Laboratory for Integrative Conservation of Plant Species with Extremely Small \\ Populations, Kunming Institute of Botany, Chinese Academy of Sciences, Kunming 650204, Yunnan, \\ China \\ 5 Department of Biology, University of Copenhagen, DK-2100 Copenhagen, Denmark \\ 6 Complete Genomics Inc., 2904 Orchard Pkwy, San Jose, CA 95134, USA \\ 7 Guangdong Provincial Key Laboratory of Genome Read and Write, Shenzhen 518083, China
}

\section{ABSTRACT}

Nyssa yunnanensis is a deciduous tree species in the family Nyssaceae within the order Cornales. As only eight individual trees and two populations have been recorded in China's Yunnan province, this species has been listed among China's national Class I protection species since 1999 and also among 120 PSESP (Plant Species with Extremely Small Populations) in the Implementation Plan of Rescuing and Conserving China's Plant Species with Extremely Small Populations (PSESP) (2011-2-15). Here, we present the draft genome assembly of N. yunnanensis. Using 10X Genomics linked-reads sequencing data, we carried out the de novo assembly and annotation analysis. The $N$. yunnanensis genome assembly is $1475 \mathrm{Mb}$ in length, containing 288,519 scaffolds with a scaffold N50 length of $985.59 \mathrm{~kb}$. Within the assembled genome, 799.51 $\mathrm{Mb}$ was identified as repetitive elements, accounting for $54.24 \%$ of the sequenced genome, and a total of 39,803 protein-coding genes were predicted.

With the genomic characteristics of $N$. yunnanensis available, our study might facilitate future conservation biology studies to help protect this extremely threatened tree species.

Published by GigaScience Press.

Preprint submitted at https://doi.org/ 10.20944/preprints202005.0417.v1

Subjects Genetics and Genomics, Plant Genetics, Botany

This is an Open Access article distributed under the terms of the Creative Commons Attribution License (http://creativecommons.org/ licenses/by/4.0/), which permits unrestricted reuse, distribution, and reproduction in any medium, provided the original work is properly cited.

Gigabyte, 2020, 1-11

\section{DATA DESCRIPTION}

Nyssa yunnanensis (NCBI: txid161873), which belongs to the family Nyssaceae, is an extremely threatened range-restricted tree species among the Critically Endangered (CR) in the IUCN Red List of Threatened Species [1], as well as a national key protected species under Class I protection in China [2]. Nyssa yunnanensis is also listed as one of 120 PSESP 


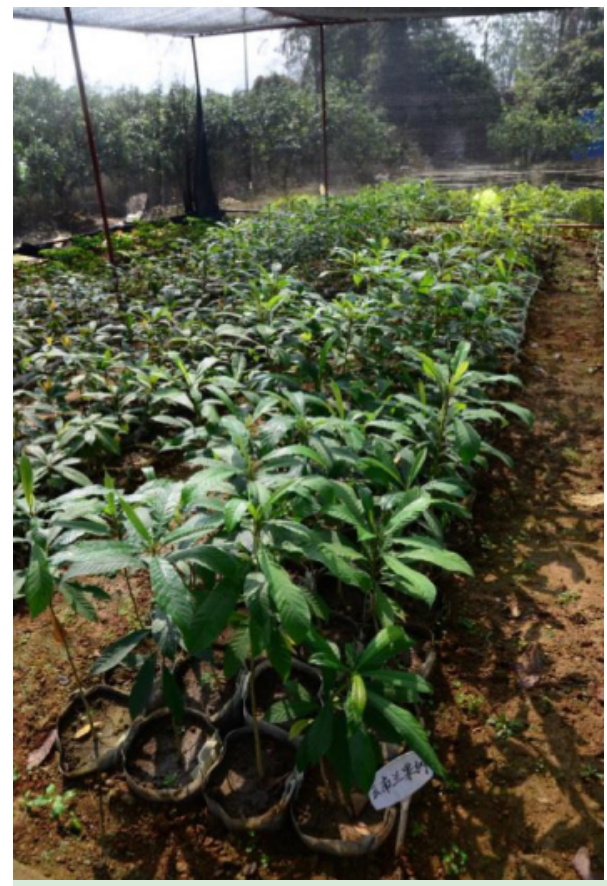

Figure 1. Photograph of Nyssa yunnanensis from Ruili, Yunnan Province, China.

(Plant Species with Extremely Small Populations) in the Implementation Plan of Rescuing and Conserving China's Plant Species with extremely Small Populations (PSESP) (2011-2-15) and as critically endangered in the Threatened Species List of China's Higher Plants [3, 4] (Figure 1). It is a canopy tree species able to reach 30 meters in height and it is functionally dioecious, consisting of two individual species. One species bears staminate flowers while the other type bears flowers that are morphologically normal but produce inaperturate and inviable pollen grains. Nyssa yunnanensis does not appear to exhibit parthenogenesis [5]. A survey of the population and ecological characteristics of $N$. yunnanensis suggested that this species is at high risk of extinction, as only two natural populations and eight individual trees have been recorded in Yunnan, China. This scarcity might be caused by both ecological and human factors [6]. In 2009, an integrated PSESP conservation strategy was initiated for $N$. yunnanensis. After more than seven years of implementation, the natural populations are now securely protected. Along with the development of propagation technologies and production of vigorous seedlings, three new populations as well as four ex situ germplasm collections of $N$. yunnanensis have now been established [7]. Phylogenetic studies of the six Nyssa species ( $N$. yunnanensis, $N$. javanica, $N$. sinensis, $N$. shangszeensis, $N$. shweliensis and $N$. wenshanensis) recognized by the Flora of China have been conducted. Based on morphological and molecular evidence, the results suggest that only $N$. sinensis, $N$. yunnanensis and $N$. javanica should be recognized as species [8].

Recent advances in whole genome sequencing technology have provided valuable genomic resources to help us better understand the origin and evolutionary history of endangered species and to improve conservation strategies [9]. Acer yangbiense, another plant species with extremely small populations endemic to Yunnan Province, was sequenced in 2019. The A. yangbiense genome has a total length of $666 \mathrm{Mb}$ with 13 


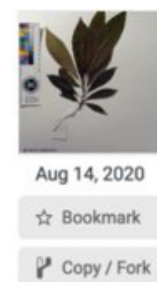

Protocols for "The draft genome assembly of the critically endangered Nyssa yunnanensis, a plant species with extremely small populations endemic to Yunnan Province, China" v

Weixue Mu', Jinpu Wei', Ting Yang', Yannan Fan', Le Cheng ${ }^{2}$, Jinlong Yang ${ }^{2}$, Ranchang Mư ${ }^{3}$, Jie Liư ${ }^{3}$, Jianming Zhao ${ }^{3}$, Weibang Sun ${ }^{4}$, Xun Xu', Xin Liu', Radoje Drmanac', Huan Liu' 'State Key Laboratory of Agricultural Genomics, BGI-Shenzhen, Shenzhen 518083, China;

${ }^{2}$ BGI-Yunnan, BGI-Shenzhen, Kunming, 650106, China; ${ }^{3}$ Forestry Bureau of Ruili, Yunnan Dehong, Ruili 678600, China:

4Yunnan Key Laboratory for Integrative Conservation of Plant Species with Extremely Small Populations, Kunming Institute of Botan y. Chinese Academy of Sciences, Kunming 650204, Yunnan, China

Works forme dx.dol.org/10.17504/protocols. io.bja6kmze

Figure 2. Protocol collection for the draft genome assembly of the critically endangered Nyssa yunnanensis, a plant species with extremely small populations endemic to Yunnan Province, China. https://www.protocols.io/ widgets/doi?uri=dx.doi.org/10.17504/protocols.io.bjq6kmze

chromosomes and a scaffold N50 size of $45 \mathrm{Mb}$ [10]. The recently published genomes of Nyssa sinensiss and Camptotheca acuminata are the only two genome assemblies that have been sequenced within the Nyssaceae family. The $N$. sinensiss genome is $1,001.42 \mathrm{Mb}$ in length with an $\mathrm{N} 50$ scaffold size of $3.62 \mathrm{Mb}$ [11], and the C. acuminate genome is $403.17 \mathrm{Mb}$ in length with an N50 scaffold size of $1753 \mathrm{~Kb}$ [12].

Although N. yunnanensis is not the first species sequenced in the Nyssaceae family, a detailed understanding of this endangered species' genomic makeup along with other information, such as population structure and reproductive biology, is urgently required to improve the PSESP conservation strategy for its continued survival.

\section{METHODS}

A protocol collection gathering together methods for DNA extraction and with DNBSEQ-G50 and 10X library construction and sequencing is available via protocols.io (Figure 2).

\section{Plant material}

We selected and sampled a $70 \mathrm{~cm}$ high individual tree of Nyssa yunnanensis from Ruili, Yunnan province, China ( $97^{\circ} 56^{\prime} 20.99^{\prime \prime} \mathrm{N}, 24^{\circ} 03^{\prime} 02.72^{\prime \prime}$ E, altitude $843 \mathrm{~m}$ ). Fresh young leaves were collected then immediately transferred into liquid nitrogen and stored in dry ice until DNA and RNA extraction. Voucher specimens and images were collected and stored in the CNGB herbarium (Figure 3). The extracted DNA is now stored in the BGI-sample center (voucher RL0289 and RL1182).

\section{DNA extraction and sequencing}

Total genomic DNA was extracted from leaf tissues of $N$. yunnanensis using a modified CTAB method [13]. Quality control was done using a NanoDrop One UV-Vis spectrophotometer (Thermo Fisher Scientific, USA) and a Qubit 3.0 Fluorometer (Thermo Fisher Scientific, USA). A Sage Science Pippin Pulse electrophoresis system was used to evaluate the molecular weight of the DNA and high-molecular-weight (HMW) gDNA with a length of around $50 \mathrm{~kb}$ was obtained for further sequencing. The HMW gDNA was then loaded onto a Chromium Controller chip with 10X Chromium reagents and gel beads, and the rest of the library preparation procedures were carried out according to the manufacturer's protocol [14]. Subsequently, the sequencing was performed on a DNBSEQ-G50 (previously known as BGISEQ-500, RRID:SCR_017979) platform at BGI-Shenzhen (BGI Co. Ltd., Shenzhen, China) according to the manufacturer's instructions [15]. Using the whole-genome shotgun 


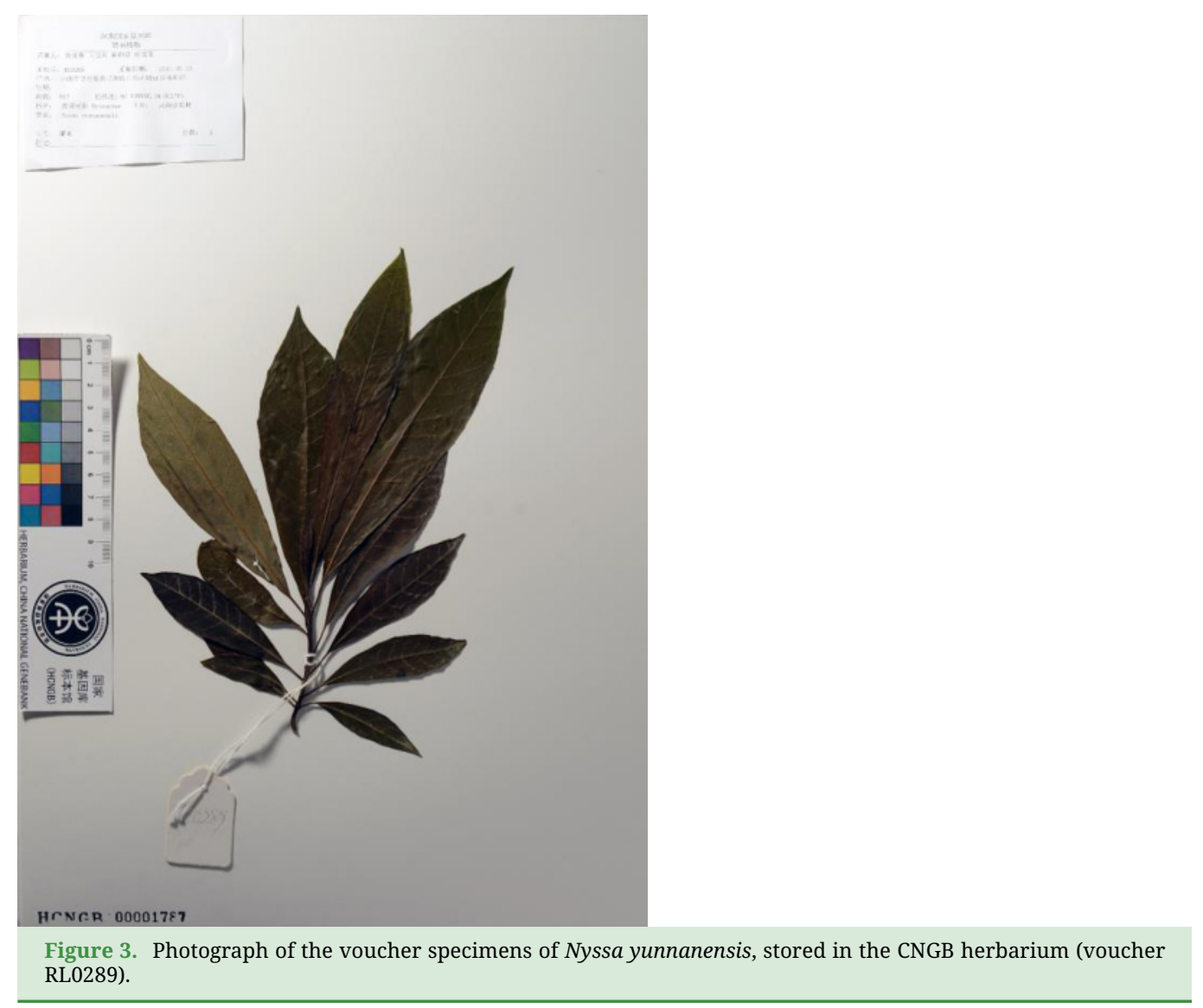

sequencing strategy, a total of $\sim 163.66 \mathrm{~Gb}$ of raw data (150 bp, paired-end) was eventually generated, covering about $100 \times$ the sequencing depth of the $1.64 \mathrm{~Gb}$ estimated genome size. All of the newly generated raw 10X Genomics reads were trimmed and filtered for adapter sequences and low-quality reads using Trimmomatic v. 0.38 (Trimmomatic, RRID:SCR_011848) [16] with the parameters "ILLUMINACLIP:adapter.fa:2:35:4 HEADCROP:5 LEADING:3 TRAILING:3 SLIDINGWINDOW:5:15 MINLEN:50”.

\section{RNA extraction and sequencing}

Total RNA was extracted from young leaves of the same individual $N$. yunnanensis tree using a CTAB-pBIOZOL method [17]. The purity, concentration, and integrity of RNA samples were measured on a NanoDrop One UV-Vis spectrophotometer (Thermo Fisher Scientific, USA), a Qubit 3.0 Fluorometer (Thermo Fisher Scientific, USA) and with Agilent 2100 Bioanalyzer on-chip electrophoresis (Agilent Technologies, Inc; Santa Clara, CA) [18], respectively, to ensure that the samples qualified for transcriptome sequencing. The cDNA library was prepared using the TruSeq RNA Sample Preparation Kit v2 (Illumina, San Diego, CA, USA), and then sequenced on a DNBSEQ-G50 platform at BGI-Shenzhen (BGI Co. Ltd., Shenzhen, China), resulting in $\sim 8.85 \mathrm{~Gb}$ of raw transcriptome data (150 bp, paired-end). All of the raw sequence reads were further filtered using SOAPfilter v2.2 (SOAP, RRID:SCR_000689) with the parameters "-y -q 33 -i 200 -g 1 -M 2 -Q 20" to remove the adapters and low-quality reads. 


\section{Genome size estimation}

The $1.64 \mathrm{~Gb}$ genome size of $N$. yunnanensis was estimated using the $21 \mathrm{k}$-mer counts of clean reads from the 10X Genomics library. First, K-mer frequency distribution analyses were performed using the kmer_freq_hash software within the gce v1.0.0 package (GCE, RRID:SCR_017332) based on the clean 10X Genomics data with the parameters "-k 21 -l reads.lst - $t$ 8". Then, gce software within the same package was used to estimate the overall characteristics of the genome, including genome size, repeat proportions, and level of heterozygosity [19].

\section{De novo genome assembly}

De novo assembly was carried out using Supernova v2.0.0 software (Supernova assembler, RRID:SCR_016756) [20] with the "-lanes $=1$-localcores $=48$-localmem $=350$-maxreads 691040000” parameters. Raw 10X Genomics linked read data without trimming were used as the software recommended. Then, the gaps within the scaffolds were filled by GapCloser v1.12 (GapCloser, RRID:SCR_015026) [21] with the parameters “-l 150 -t 32" based on the clean 10X Genomics pair-end reads.

\section{Genome evaluation}

The completeness of the $N$. yunnanensis assembly was estimated using two strategies. First, we assessed completeness using BUSCO (BUSCO; v3.0.2, RRID:SCR_015008) [22] with the parameters “-l Embryophyta_odb10 -m geno -c 8 -f”. Second, all the clean transcriptome reads and clean $10 \mathrm{X}$ Genomics reads were mapped back to the final genome assembly using BWA-MEM (BWA, version 0.7.16, RRID:SCR_010910) [23] with default parameters.

\section{Repeat annotation}

Repetitive elements were identified using both homology-based and de novo predictions in the $N$. yunnanensis genome assembly. For homology-based prediction, RepeatMasker v3.3.0 (RepeatMasker, RRID:SCR_012954) and RepeatProteinMasker v3.3.0 [24] were applied with parameters "-nolow -no_is -norna -engine ncbi -parallel 1 -lib RepeatMaskerLib.embl.lib" and “-engine ncbi -noLowSimple -pvalue 0.0001", respectively. The N. yunnanensis genome sequence was aligned against the known repeats database, Repbase v16.10 [25], at both DNA and protein levels to identify the known repetitive elements. For de novo prediction, RepeatModeler v1.0.5 (RepeatModeler, RRID:SCR_015027) [26] was first executed to build a de novo repeat library using the $N$. yunnanensis genome assembly with parameters "-engine ncbi -name mydb”. Then, RepeatMasker v3.3.0 [24] was employed to align the $N$. yunnanensis genome sequences against the de novo repeat library with parameters "-nolow -no_is -norna -engine ncbi -parallel 1 -lib final.library" to identify the repetitive elements. LTR_FINDER v1.05 (LTR_Finder, RRID:SCR_015247) [27] was used with parameters "-w 2 -s Athal-tRNAs.fa" for $a b$ initio LTR retrotransposon finding and Tandem Repeats Finder v4.07 [28] was used with parameters “2 778010502000 -d -h" to identify tandem repeats.

\section{Gene prediction}

The $N$. yunnanensis genome with repetitive regions masked was used to predict more genes. Protein-coding genes were predicted based on de novo prediction, homology search, and RNA evidence. For de novo prediction, Genemark-ES v4.21 (GeneMark, 
RRID:SCR_011930) [29] was used to carry out self-training with the default settings. To search for homologs, protein sequences of Camptotheca acuminate and Arabidopsis thaliana were used as references. For RNA evidence, a de novo approach was used. All of the clean RNA reads were assembled into inchworm contigs to function as expressed sequence tag evidence using Trinity v2.0.6 (Trinity, RRID:SCR_013048) [30] with the parameters “-min_contig_length 100 -min_kmer_cov 2 -inchworm_cpu 6 -group_pairs_distance 200 -no_run_chrysalis”. MAKER-P v2.31 (MAKER, RRID:SCR_005309) [31] was used to perform the prediction based on the evidence above. The first round of MAKER-P was run with the "protein2genome" and "est2genome" parameter set to "1" to obtain evidence-supported gene models. SNAP [32] was then applied to train these gene models. Then, MAKER-P was run for the second round with default parameters to generate the final consensus gene set. The search tool tRNAscan-SE v1.23 (tRNAscan-SE, RRID:SCR_010835) [33] was used for identifying tRNA genes. The rRNA sequences of Arabidopsis thaliana and Oryza sativa were BLAST against the $N$. yunnanensis assembly using BLASTN (BLASTN, RRID:SCR_001598) (E-value $\leq 1 \mathrm{e}-05$ ) to identify rRNA genes. MicroRNAs and snRNAs were detected by searching the sequences against the Rfam database [34] using INFERNAL (Infernal, RRID:SCR_011809) [35] software.

\section{Functional annotation}

The predicted gene models were further functionally annotated by querying the protein sequences against those in the public databases of Swiss-Prot [36], NCBI non-redundant (NR), KEGG [37], and TrEMBL with BLASTP (BLASTP, RRID:SCR_001010) with the parameters “-e 1e-05 -a 5 -m 8 -F F”. InterProScan v5.21 (InterProScan, RRID:SCR_005829) [38] was further used to search for the protein motifs and domains against public domain databases including the PFAM, PANTHER, PRINTS, PROSITE, ProDom, and SMART databases with the parameters "-goterms -f tsv -appl Pfam -appl PRINTS -appl ProSiteProfiles -appl ProSitePatterns -appl ProDom -appl SMART”.

\section{RESULTS \& DISCUSSION}

\section{Assembly and annotation of the N. yunnanensis genome}

We assembled the draft genome assembly of the highly endangered tree species $N$. yunnanensis with DNBSEQ-G50 data from a 10X Genomics linked-reads library. The final genome assembly was $1.475 \mathrm{~Gb}$ in length, which is close to the estimated genome size of $1.64 \mathrm{~Gb}$, with a scaffold $\mathrm{N} 50$ of $985.59 \mathrm{~Kb}$ and a contig N50 of $32.33 \mathrm{~Kb}$ (Table 1). The $N$. yunnanensis genome size we assembled was also close to the estimated genome size of $1.23 \mathrm{~Gb}$ based on the raw data produced [39] for the Digitization of the Ruili Botanical Garden project [40]. The GC content of the $N$. yunnanensis assembly was $42.18 \%$ excluding gaps, and a total of $54.24 \%$ of the assembly was composed of repetitive elements (Table 2). We ultimately obtained 39,803 protein-coding genes and successfully annotated $96.57 \%$ of the $N$. yunnanensis gene loci (Table 3). Non-coding genes were also annotated, identifying 175 microRNA (miRNA), 1,130 transfer RNA (tRNA), 1,502 ribosomal RNA (rRNA) and 3,106 small nuclear RNA (snRNA) genes (Table 4).

\section{Data validation and quality control}

The BUSCO analysis showed that up to 1244 (90.5\%) of the expected 1375 conserved plant orthologs were detected as complete in the $N$. yunnanensis assembly and $81.9 \%$ of them 


\begin{tabular}{|c|c|c|c|c|}
\hline \multirow[t]{2}{*}{ Parameters } & \multicolumn{2}{|c|}{ Scaffold } & \multicolumn{2}{|c|}{ Contig } \\
\hline & Length (bp) & Number & Length (bp) & Number \\
\hline Maximal length (bp) & $17,928,324$ & & 636,521 & \\
\hline N90 & 3,639 & 38,762 & 2,907 & 67,003 \\
\hline N80 & 8,911 & 13,177 & 6,795 & 37,603 \\
\hline N70 & 27,779 & 2,699 & 12,643 & 22,971 \\
\hline N60 & 308,718 & 527 & 21,401 & 14,910 \\
\hline N50 & 985,593 & 280 & 32,329 & 9,853 \\
\hline $\mathrm{N} 40$ & $1,699,409$ & 166 & 4,5737 & 6,370 \\
\hline N30 & $2,587,311$ & 96 & 62,625 & 3,875 \\
\hline $\mathrm{N} 20$ & $3,773,602$ & 49 & 86,821 & 2,057 \\
\hline N10 & $6,183,248$ & 17 & 127,405 & 771 \\
\hline Total length (bp) & $1,474,960,449$ & & $1,330,978,457$ & \\
\hline Number $\geq 100 \mathrm{bp}$ & & 288,519 & & 320,818 \\
\hline Number $\geq 2000 b p$ & & 57,709 & & 79,919 \\
\hline $\begin{array}{l}\text { Percentage of } \mathrm{N} \\
\text { content }\end{array}$ & & $9.76 \%$ & & \\
\hline
\end{tabular}

Table 2. Statistics of repetitive sequences identified in the $N$. yunnanensis genome.

\begin{tabular}{ccc}
\hline Category & Total repeat length (bp) & \% of assembly \\
DNA & $133,291,367$ & $9.04 \%$ \\
LINE & $47,813,283$ & $3.24 \%$ \\
SINE & $1,053,993$ & $0.07 \%$ \\
LTR & $690,959,337$ & $46.87 \%$ \\
Tandem repeats & 6,108 & $0.0004 \%$ \\
Unknown & 135 & $0.000009 \%$ \\
\hline Combined & $799,507,629$ & $54.24 \%$ \\
\hline Note: DNA: DNA transposon; LINE: long interspersed nuclear element; SINE: short interspersed nuclear elements; \\
LTR: long terminal repeat.
\end{tabular}

Table 3. Summary of protein-coding genes annotated in the N. yunnanensis genome.

\begin{tabular}{ll} 
Characteristics of protein-coding genes & \\
Total number of protein-coding genes & 39,803 \\
Mean gene size (bp) & 2576.05 \\
Mean CDS length (bp) & 957.64 \\
Mean exon number per gene & 4.16 \\
Mean exon length (bp) & 230.26 \\
Mean intron length (bp) & 512.32 \\
Functional annotation by searching public databases & \\
\% of proteins with hits in Swiss-Prot database & 76.01 \\
\% of proteins with hits in NCBI nr database & 96.30 \\
\% of proteins with hits in KEGG database & 72.15 \\
\% of proteins with hits in TrEMBL database & 95.90 \\
\% of proteins with hits in Interpro database & 70.99 \\
\% of proteins with functional annotation (combined) & 96.57 \\
\hline
\end{tabular}

were identified as complete and single-copy genes (Table 5). The RNA mapping showed that $98.95 \%$ of the reads could be successfully mapped back to the assembled genome and $83.74 \%$ of them were properly paired. The DNA mapping resulted in a $96.88 \%$ mapping rate and $86.74 \%$ of them were properly paired. These results demonstrate the high completeness of the $N$. yunnanensis assembled genome. 


\begin{tabular}{|c|c|c|c|c|c|}
\hline Type & & Number & $\begin{array}{l}\text { Average length } \\
\text { (bp) }\end{array}$ & Total length (bp) & $\%$ of genome \\
\hline miRNA & & 175 & 120.8629 & 21,151 & 0.0014 \\
\hline tRNA & & 1,130 & 75.2221 & 85,001 & 0.0058 \\
\hline \multirow[t]{5}{*}{ rRNA } & rRNA & 1,502 & 142.5366 & 214,090 & 0.0145 \\
\hline & $18 S$ & 469 & 231.5117 & 108,579 & 0.0074 \\
\hline & $28 S$ & 458 & 106.0764 & 48,583 & 0.0033 \\
\hline & $5.8 \mathrm{~S}$ & 116 & 111.0603 & 12,883 & 0.0009 \\
\hline & $5 S$ & 459 & 95.9586 & 44,045 & 0.0030 \\
\hline \multirow[t]{4}{*}{ snRNA } & snRNA & 3,106 & 108.3973 & 336,682 & 0.0228 \\
\hline & CD-box & 2,911 & 106.8636 & 311,080 & 0.0211 \\
\hline & HACA-box & 51 & 129.3333 & 6,596 & 0.0004 \\
\hline & splicing & 144 & 131.9861 & 19,006 & 0.0013 \\
\hline
\end{tabular}

\begin{tabular}{|lrr|}
\hline Table 5. BUSCO assessment of $N$. yunnanensis genome. & & \\
\hline BUSCO benchmark & Number of genes & Percentage \\
Complete BUSCOs & 1244 & 90.5 \\
Complete and single-copy BUSCOs & 1126 & 81.9 \\
Complete and duplicated BUSCOs & 118 & 8.6 \\
Fragmented BUSCOs & 63 & 4.6 \\
Missing BUSCOs & 68 & 4.9 \\
Total & 1375 & $/$ \\
\hline
\end{tabular}

\section{Potential for reuse}

Here we report a draft genome assembly of the PSESP plant species $N$. yunnanensis. The completeness assessment carried out by reads mapping and BUSCO assessment indicated the high completeness of this draft assembly. As part of the 10KP (10,000 Plants) Genome Sequencing Project [41], the sequencing data and the well-annotated draft assembly generated in this study can be used for future phylogenetics and comparative genomics analyses, such as resolving the controversial phylogenetic relationships within the Nyssa genus. In particular, due to the extremely small population structure of $N$. yunnanensis, the genomic resources released in this study will support further research on the conservation biology of this highly endangered species as well as other PSESP species.

\section{ABBREVIATIONS}

10KP: 10,000 plant genome project; BUSCO: Benchmarking Universal Single-Copy Orthologs; CR: Critically Endangered; CTAB: cetyl-triethylammonium bromide; HMW:

high-molecular-weight; PSESP: Plant species with extremely small populations;

\section{AVAILABILITY OF SUPPORTING DATA}

The 10X Genomics clean reads and RNA-seq clean reads are deposited in NCBI under the BioProject accession PRJNA438407, with SRA accession number SRX8345787 and SRX8373586. These reads are also deposited in the CNGB Nucleotide Sequence Archive (CNSA) with accession number CNP0001048. Genome assembly, protein-coding genes, and repeat annotations are deposited in the GigaScience GigaDB repository [42].

\section{FUNDING}

This work was supported by funding from the National Key R\&D Program of China (Grant No. 2019YFC1711000), the Shenzhen Municipal Government of China (Grant No. 
JCYJ20170817145512476), the Shenzhen Municipal Government of China Peacock Plan (No. KQTD2015033017150531), the Guangdong Provincial Key Laboratory of Genome Read and Write (Grant No. 2017B030301011), and the Yunnan Innovation Team Program for conservation and utilization of PSESP(Plant Species with Extremely Small Populations) Ggrant No. 2019HC015). This work was also supported by the China National GeneBank.

\section{COMPETING INTERESTS}

The authors W.M., J.W., T.Y., Y.F., L.C., J.Y., X.X., X.L., R.D. and H.L. are BGI-Shenzhen employees.

\section{AUTHOR CONTRIBUTIONS}

H.L., R.D., W.S., X.L. and X.X. conceived and supervised the study; J.W., L.C., J.Y., R.M., J.L. and J.Z. prepared the samples; W.M., Y.F. and T.Y. analyzed the results; W.M. wrote the manuscript with input from all authors. All authors read and approved the final manuscript.

\section{SUBJECT AREAS}

Genetics and Genomics; Plant Genetics, Botany

\section{REFERENCES}

1 IUCN Red List of Threatened Species. http://www.iucnredlist.org/.

2 List of National Key Protected Wild Plants. http://www.forestry.gov.cn/yemian/minglu1.htm.

3 Sun WB, Yang J, Dao Z, Study and Conservation of Plant Species with Extremely Small Population (PSESP) in Yunnan Province, China. Beijing, China: Science Press 2019.

4 Yang J, Cai L, Liu D, Chen G, Gratzfeld J, Sun W, China's conservation program on Plant Species with Extremely Small Populations (PSESP): Progress and perspectives. Biological Conservation, 2020; 244, doi:10.1016/j.biocon.2020.108535.

5 Sun B-L, Zhang C-Q, Lowry PP, Wen J, Cryptic Dioecy in Nyssa Yunnanensis (Nyssaceae), A Critically Endangered Species from Tropical Eastern Asia. Annals of the Missouri Botanical Garden, 2009; 96(4): 672-84, 13.

6 Chen W, Shi F, Yang W, Zhou Y, Chen H, Population status and ecological characteristics of Nyssa yunnanensis. Journal of Northeast Forestry University, 2011; 39(9): 17-9, 61.

7 Yang W-Z, Zhang S-S, Wang W-B, Kang H-M, Ma N, A sophisticated species conservation strategy for Nyssa yunnanensis, a species with extremely small populations in China. Biodiversity and Conservation, 2017; 26(4): 967-81, doi:10.1007/s10531-016-1282-8.

8 Wang N, Milne RI, Jacques FMB, Sun B-L, Zhang C-Q, Yang J-B, Phylogeny and a revised classification of the Chinese species of Nyssa (Nyssaceae) based on morphological and molecular data. TAXON, 2012; 61(2): 344-54, doi:10.1002/tax.612006.

9 Supple MA, Shapiro B, Conservation of biodiversity in the genomics era. Genome Biol., 2018; 19(1): 131, doi:10.1186/s13059-018-1520-3.

10 Yang J, Wariss HM, Tao L, Zhang R, Yun Q, Hollingsworth $\mathbf{P}$ et al. De novo genome assembly of the endangered Acer yangbiense, a plant species with extremely small populations endemic to Yunnan Province, China. Gigascience, 2019; 8(7): doi:10.1093/gigascience/giz085.

11 Yang X, Kang M, Yang Y, Xiong H, Wang M, Zhang Z et al. A chromosome-level genome assembly of the Chinese tupelo Nyssa sinensis. Sci. Data, 2019; 6(1): 282, doi:10.1038/s41597-019-0296-y.

12 Zhao D, Hamilton JP, Pham GM, Crisovan E, Wiegert-Rininger K, Vaillancourt B et al. De novo genome assembly of Camptotheca acuminata, a natural source of the anti-cancer compound camptothecin. Gigascience, 2017; 6(9): 1-7, doi:10.1093/gigascience/gix065.

13 Sahu SK, Thangaraj M, Kathiresan K, DNA Extraction Protocol for Plants with High Levels of Secondary Metabolites and Polysaccharides without Using Liquid Nitrogen and Phenol. ISRN Mol. Biol., 2012; 2012: 205049. doi:10.5402/2012/205049. 
14 Etherington GJ, Heavens D, Baker D et al. 10x Genomics Library Construction. protocols.io 2020; https://doi.org/10.17504/protocols.io.bd3vi8n6.

15 Huang J, Liang X, Xuan Y et al. BGISEQ-500 WGS library construction. protocols.io 2018; https://doi.org/10.17504/protocols.io.ps5dng6.

16 Bolger AM, Lohse M, Usadel B, Trimmomatic: a flexible trimmer for Illumina sequence data. Bioinformatics, 2014; 30(15): 2114-20, doi:10.1093/bioinformatics/btu170.

17 Edmunds S, RNA extraction for plant samples using CTAB-pBIOZOL. protocols.io 2017; https://doi.org/10.17504/protocols.io.gsnbwde.

18 Simbolo M, Gottardi M, Corbo V, Fassan M, Mafficini A, Malpeli G et al. DNA qualification workflow for next generation sequencing of histopathological samples. PLoS One, 2013; 8(6): e62692. doi:10.1371/journal.pone.0062692.

19 Liu B, Shi Y, Yuan J, Hu X, Zhang H, Li N et al. Estimation of genomic characteristics by analyzing k-mer frequency in de novo genome projects. 2013; arXiv preprint arXiv:1308.2012v2.

20 Weisenfeld NI, Kumar V, Shah P, Church DM, Jaffe DB, Direct determination of diploid genome sequences. Genome Res., 2017; 27(5): 757-67, doi:10.1101/gr.214874.116.

21 Luo R, Liu B, Xie Y, Li Z, Huang W, Yuan J et al. SOAPdenovo2: an empirically improved memory-efficient short-read de novo assembler. Gigascience, 2012; 1(1): 18, doi:10.1186/2047-217X-1-18.

22 Seppey M, Manni M, Zdobnov EM, BUSCO: Assessing Genome Assembly and Annotation Completeness. Methods Mol. Biol., 2019; 1962: 227-45, doi:10.1007/978-1-4939-9173-0_14.

23 Li H, Aligning sequence reads, clone sequences and assembly contigs with BWA-MEM. 2013; arXiv preprint arXiv:1303.3997v2.

24 Tarailo-Graovac M, Chen N, Using RepeatMasker to identify repetitive elements in genomic sequences. Curr. Protoc. Bioinformatics, 2009; Chapter 4: Unit 4 10; doi:10.1002/0471250953.bi0410s25.

25 Jurka J, Kapitonov VV, Pavlicek A, Klonowski P, Kohany O, Walichiewicz J, Repbase Update, a database of eukaryotic repetitive elements. Cytogenet. Genome Res., 2005; 110(1-4): 462-7, doi:10.1159/000084979.

26 Price AL, Jones NC, Pevzner PA, De novo identification of repeat families in large genomes. Bioinformatics, 2005; 21(Suppl 1): i351-8, doi:10.1093/bioinformatics/bti1018.

$27 \mathrm{Xu}$ Z, Wang H, LTR_FINDER: an efficient tool for the prediction of full-length LTR retrotransposons. Nucleic Acids Res., 2007; 35(Web Server issue): W265-8, doi:10.1093/nar/gkm286.

28 Benson G, Tandem repeats finder: a program to analyze DNA sequences.. Nucleic Acids Res., 1999; 27(2): 573-80, doi:10.1093/nar/27.2.573.

29 Lomsadze A, Ter-Hovhannisyan V, Chernoff YO, Borodovsky M, Gene identification in novel eukaryotic genomes by self-training algorithm. Nucleic Acids Res., 2005; 33(20): 6494-506, doi:10.1093/nar/gki937.

30 Haas BJ, Papanicolaou A, Yassour M, Grabherr M, Blood PD, Bowden J et al. De novo transcript sequence reconstruction from RNA-seq using the Trinity platform for reference generation and analysis. Nat. Protoc., 2013; 8(8): 1494-512, doi:10.1038/nprot.2013.084.

31 Campbell MS, Holt C, Moore B, Yandell M, Genome Annotation and Curation Using MAKER and MAKER-P. Curr. Protoc. Bioinformatics, 2014; 48: 411 1-39, doi:10.1002/0471250953.bi0411s48.

32 Korf I, Gene finding in novel genomes. BMC Bioinformatics, 2004; 5: 59, doi:10.1186/1471-2105-5-59.

33 Lowe TM, Eddy SR, tRNAscan-SE: a program for improved detection of transfer RNA genes in genomic sequence. Nucleic Acids Res., 1997; 25(5): 955-64, doi:10.1093/nar/25.5.955.

34 Nawrocki EP, Burge SW, Bateman A, Daub J, Eberhardt RY, Eddy SR et al. Rfam 12.0: updates to the RNA families database. Nucleic Acids Res., 2015; 43(Database issue): D130-7, doi:10.1093/nar/gku1063.

35 Nawrocki EP, Kolbe DL, Eddy SR, Infernal 1.0: inference of RNA alignments. Bioinformatics, 2009; 25(10): 1335-7, doi:10.1093/bioinformatics/btp157.

36 Bairoch A, Apweiler R, The SWISS-PROT protein sequence database and its supplement TrEMBL in 2000. Nucleic Acids Res., 2000; 28(1): 45-8, doi:10.1093/nar/28.1.45.

37 Kanehisa M, Goto S, KEGG: kyoto encyclopedia of genes and genomes. Nucleic Acids Res., 2000; 28(1): 27-30, doi:10.1093/nar/28.1.27. 
38 Quevillon E, Silventoinen V, Pillai S, Harte N, Mulder N, Apweiler R et al. InterProScan: protein domains identifier. Nucleic Acids Res., 2005; 33(Web Server issue): W116-20, doi:10.1093/nar/gki442.

39 Liu H, Wei J, Yang T, Mu W, Song B, Yang T, Fu Y, Wang X, Hu G, Li W, Zhou H, Chang Y, Chen X, Chen H, Cheng L, He X, Cai H, Cai X, Wang M, Li Y, Yang J, Wang Y, Mu R, Liu J, Zhao J, Huang Z, Liu X, Genomic data of Nanmaohu Park vascular plant specimen, RL0289. GigaScience Database, 2019; https://doi.org/10.5524/101352.

40 Liu H, Wei J, Yang T, Mu W, Song B, Yang T et al. Molecular digitization of a botanical garden: high-depth whole-genome sequencing of 689 vascular plant species from the Ruili Botanical Garden. Gigascience, 2019; 8(4): doi:10.1093/gigascience/giz007.

41 Cheng S, Melkonian M, Smith SA, Brockington S, Archibald JM, Delaux PM et al. 10KP: A phylodiverse genome sequencing plan. Gigascience, 2018; 7(3): 1-9, doi:10.1093/gigascience/giy013.

42 Mu W, Wei J, Yang T, Fan Y, Cheng L, Yang J, Mu R, Liu J, Zhao J, Sun W, Xu X, Liu X, Drmanac R, Liu H, Genome data for the draft assembly of the Chinese tertiary relict tree, Nyssa yunnanensis. GigaScience Database, 2020; http://doi.org/10.5524/100752. 